Adam et al. MKH (2022). 48-52

DOI: $10.20473 / \mathrm{mkh} . \mathrm{v} 33 \mathrm{i1} .2022 .48-52$

\title{
Traumatic Ventriculitis in an African Ostrich: A Case Report
}

\author{
Mohammed Adam¹, Abdullateef Abiodun Ajadi1* ${ }^{*}$, Jamila Abiodun Atata1 ${ }^{1}$, \\ Olatunde Babatunde Akanbi ${ }^{1}$
}

\author{
${ }^{1}$ Department of Veterinary Pathology, University of Ilorin, Nigeria \\ *E-mail: ajadi.aa@unilorin.edu.ng
}

\begin{abstract}
Traumatic ventriculitis or hardware disease is the gizzard perforation by sharp foreign bodies (metallic or sharp object) that leads to ulcerative lesions. The present Case Report was to investigate through post mortem examination the cause of African ostrich death. Necropsy was conducted on a dead male adult African Ostrich presented to the post mortem unit of the Department of Veterinary Pathology, University of Ilorin, Ilorin, Nigeria. The gross lesions showed multifocal ecchymotic and petechial haemorrhages and lacerations on the left shank and thigh as well as the cloaca and vent of the bird. A perforation of the proventriculus with adhesion of a pointed nail-like metallic sharp object of $9.4 \mathrm{~cm}$ in length was observed. Histopathological findings revealed obliteration of the tubules and vascular congestion which predisposed the animal to metabolic disorder.
\end{abstract}

Keywords: Ventriculitis, Ostrich, Necropsy, Gizzard, Metallic

INTRODUCTION

Ostrich is the world's largest living bird, weighing over $200 \mathrm{~kg}$ with a height of about $3.0 \mathrm{~m}$. This bird belongs to the family Struthionidae and genus struthio (Cooper, 2000). The bird's dome-backed shape, its desert living conditions and old-shaped feet are reminiscent of the camel, thus the species name, camelus. Four subspecies are currently recognized; which can be found in the northern (Struthio camelus camelus), eastern (Struthio camelus massaicus, Struthio camelus molybdophanes)and southern (Struthio camelus australis) savannahs of the African continent. A hybridized ostrich, that is a combination of Struthio camelus australis, Struthio camelus massaicus and Struthio camelus syriacus is known as "African black" or "domestic" ostrich (Verwoerd, 2000). This bird is one of the largest ground-dwelling birds that share common characteristic of flightlessness. Other group members of these birds include; rhea, emu, kiwi and cassowary (Cooper, 2000). Foreign body 
Adam et al. MKH (2022). 48-52

DOI: $\underline{10.20473 / \mathrm{mkh} . v 33 i 1.2022 .48-52}$

traumatic injuries are common disorders in large ruminants reared in industrial areas. This condition could be traumatic reticulitis, traumatic reticuloperitonitis and traumatic pericarditis. The common cause of these conditions including ventriculitis is ingestion of sharp metallic foreign objects through the feed material (Prasad et al., 2017).

\section{CASE HISTORY AND SIGNALMENT}

The carcass of a three-year-old, male Ostrich weighed $200 \mathrm{~kg}$ was presented for necropsy at the Department of Veterinary Pathology, University of Ilorin. It was one of the two male ostriches in the same fenced perimeter space with a female. About four weeks before the presentation, the particular Ostrich was diarrhoeic (yellowish-white, foamy faeces). There was clinical recovery after treatment. About three weeks after the clinical recovery, there was a relapse of the diarrhoeic experience. The treatment regimen employed earlier was being repeated but the bird died some four days from the onset of the diarrhoea.

\section{NECROPSY EXAMINATION PROTOCOL}

The body of the ostrich was wet with water and examined for external parasites and to minimize flying feathers during necropsy. The ostrich was thereafter placed on necropsy table on dorsal decumbency. Each leg in-turn of the ostrich was drawn outward away from the body. The skin between the legs and abdomen on each side was incised. Both legs were then grasped firmly in the area of the femur. The legs were then bent forward, downward and outward, until the heads of both femurs are free from the acetabular attachment. This allowed both legs to freely lie flat on the table. The skin between keel and vent at a mid-way was incised. The cut edge was forcibly reflected forward, and cut through the entire ventral aspect of the body to expose the neck. The abdominal wall was thereafter cut transversely midway between the keel and vent and then through the breast muscles on each side. The rib cage, the coracoid from the body and clavicle on both sides were cut with a bone cutter. The sternum was cut opened and the attached visceral structures were removed from the abdominal cavity for gross examination.

\section{POST MORTEM FINDINGS}

The animal was in good body condition but with slightly ruffle feathers. The mucus membrane of the eyes contained straw coloured mucoid exudates. The shanks and lateral surface of the left thigh had multifocal lacerations and echymotic haemorrhages (Plate A). The cloaca and vent had multifocal ecchymotic 
Adam et al. MKH (2022). 48-52

DOI: 10.20473/mkh.v33i1.2022.48-52

haemorrhages that matted the vent feathers. There was a double pointed sharp metallic nail-like object (Plates B and C) of $9.4 \mathrm{~cm}$ long that perforated the proventricular muscular wall but did not perforate the wall. The perforated portion of the muscular layer was thickened (about $10 \mathrm{~mm}$ in diameter) hard and granulomatous. The crop was filled with ingesta and the intestines were apparently normal and filed with faeces. A farm visit was carried out to ascertain the nature of the farm, the management procedures and general well being of the one Ostrich left and other animals in the farm. Microscopically, the lesions include collapsed tubular glands, reduced lumen of tubular gland and secretory gland and vascular congestion (Plate D).
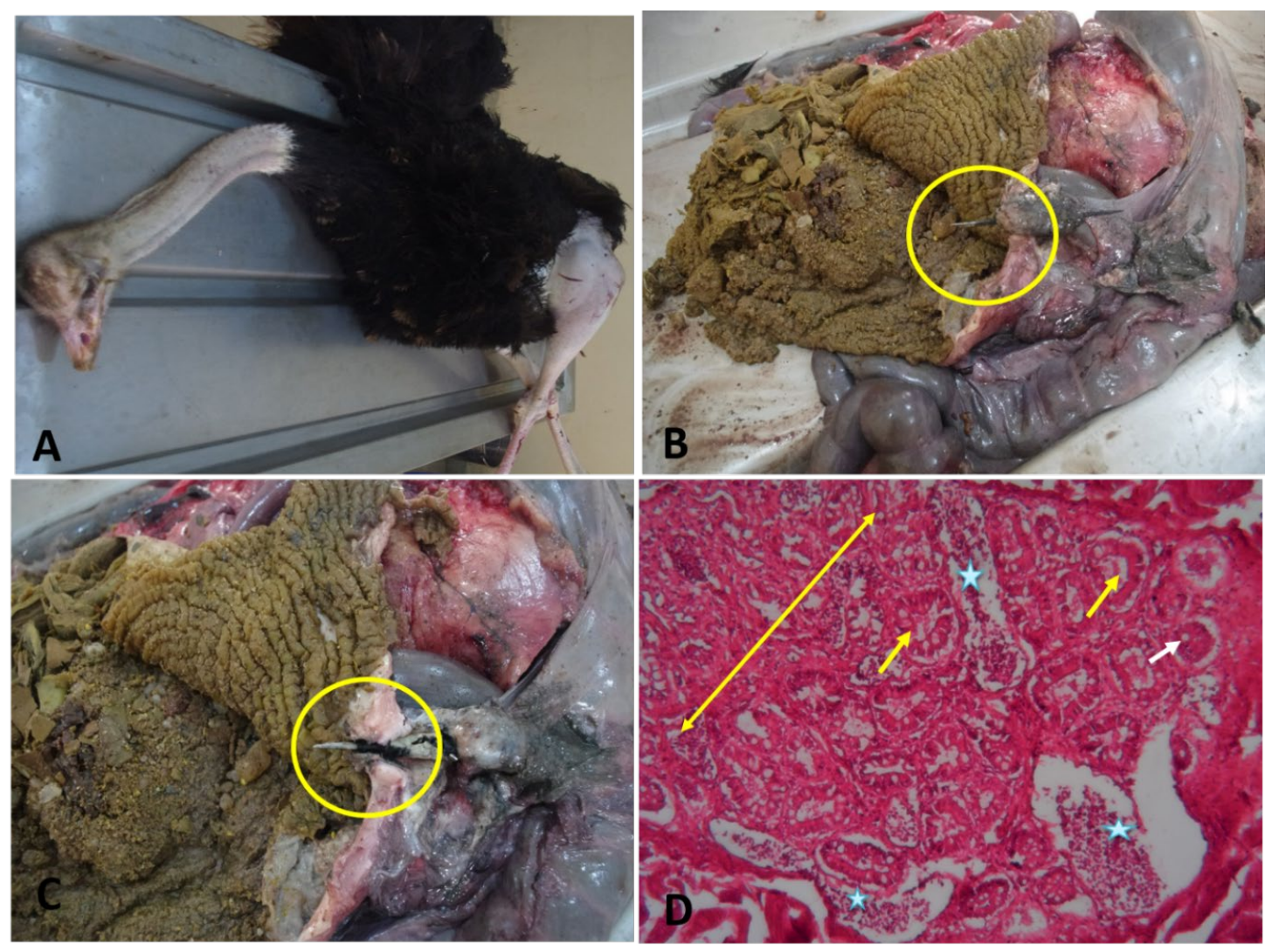

Figure 1. Plate A: showing the ostrich on necropsy table. Plate B and C: showing sharp pointed metallic object penetrated from serosa surface through mucosa layer of gizzard (yellow circle). Plate D: photomicrograph of gizzard tissue section from the ostrich showing area collapsed tubular glands (double end arrow), reduced lumen of tubular gland (yellow arrow), secretory gland (star) and vascular congestion (white arrow), H\&E x 40. 
Adam et al. MKH (2022). 48-52

DOI: $10.20473 / \mathrm{mkh} . v 33 i 1.2022 .48-52$

\section{HISTOPATHOLOGICAL EXAMINATION PROTOCOL}

After 48 hours, the fixed tissues sections of gizzard were cut into pieces of 2-3 mm thickness and dehydrated using ascending grades $(70 \%, 80 \%, 90 \%$ and $100 \%$ ) of alcohol for 15 minutes, followed by clearing in xylene and

\section{DISCUSSION}

The farm visit revealed the presence of one other Ostrich as well as other animals including broilers and cattle. Feed in the farm is manually compounded and renovation of the pens and cages had just been done recently. This afore mentioned factor can prone the animal to ingestion of metallic and sharp object that can lead to hardware injury (Al-Abbadi et al., 2014). Urbanization and lack of adequate space for the captive wild animals, many of these animals are allowed to feed and graze around the limited captive areas which make them eat anything that comes their way. Besides this, pica due to mineral deficiencies also makes the animals consume rubbish stuffs containing metallic objects leading to foreign body traumatic injury. The digestive system of ostrich (Ratites) reflects the ecological niche of these large grazing ungulates (Aganga et al., 2003). Ratites have no crops, and the large proventriculus serves as feed storage vat. The proventriculus of the ostrich is a large, embedded in paraffin wax. Sections of 4-5 micron thickness were cut and stained by hematoxylin and eosin method (Bancroft and Gamble, 2008). Finally, the stained slides were examined at $\times 4, \quad x 10$ and $\times 100$ magnifications for the presence of characteristic and/or suggestive lesions using ordinary light microscope.

dilated, thin-walled structure that extends caudally to the ventriculus (gizzard) (Aganga et al., 2003). The distal extremity of the ostrich proventriculus passes dorsal to the ventriculus and empties into this organ through a large opening on its caudal aspect. Ostrich ventriculus is a thickwalled structure similar to that in seedeating birds (Cooper, 2004). The ventriculus is located slightly to the left of the midline at the caudal border of the sternum. Though the proventriculus and ventriculus normally contain small stones, gastric impaction from the consumption of foreign bodies is a common problem in ratites, particularly in juvenile birds.

Foreign body ingestion is not an uncommon finding in captive ratites. Metallic foreign bodies (nails, wire, screws, etc.) may cause hardware disease, especially in captive facilities where construction work has been undertaken within enclosures, or where materials are brought in with leaf litter or other substrate. The powerful contractions of the gizzard muscles can easily 
Adam et al. MKH (2022). 48-52

DOI: $\underline{10.20473 / \mathrm{mkh} . v 33 i 1.2022 .48-52}$

force sharp objects through the muscular wall (Smith 2020). This causes a reduction in ventricular contraction, insufficient digestion of food and colic. Commonly, the foreign body may penetrate the proventricular wall, leading to an acute, generalised purulent peritonitis, or to a local peritonitis with abscess formation on the surface of the proventriculus or gizzard, or duodenum (Smith 2020). The ensuing colic may be responsible for self-mutilation through kicking and scratching leading to laceration and haemorrhages observed on the body of the Ostrich presented in this Case Report.

\section{CONCLUSION}

Hardware traumatic injury of the proventriculus is the cause of death in the Africa-ostrich. There is need for periodic environmental clearingof metallic and hard sharp objects to avoid or reduce risk of future occurrence hardware traumatic injury on this farm. It is also recommended that X-ray should be included in any reported case to rule out hardware traumatic injury and possible surgical intervention may be of advantage. feeding and nutrition. Pakistan Journal of Nutrition, 2(2), 60-67.

Al-Abbadi, O. S., Abu-Seida, A. M., \& Al-Hussainy, S. M. (2014). Studies on rumen magnet usage to prevent hardware disease in buffaloes. Veterinary World, 7(6).

Cooper, R. G., \& Mahroze, K. M. (2004). Anatomy and physiology of the gastro-intestinal tract and growth curves of the ostrich (Struthio camelus). Animal Science Journal, 75(6), 491-498.

Cooper, R. G. (2000). Critical factors in ostrich (Struthio camelus australis) production: a focus on southern Africa. World's Poultry Science Journal, 56(3), 248-265.

Devi Prasad, V., Ravi Kumar, P., Harikrishna, N.V.V. and Bhagyaraju, D. (2017). Traumatic reticulitis, reticulo-peritonitis and pericarditis (Foreign body syndrome) in bovines Journal of Livestock Science, 8: 98-102.

Smith, D. A. (2020). Ratites. HandRearing Birds, 75-88.

Verwoerd, D. J. (2000). Ostrich diseases. Revue Scientifique et TechniqueOffice International des Epizooties, 19(2), 638-652.

\section{REFERENCE}

Aganga, A. A., Aganga, A. O., \& Omphile, U. J. (2003). Ostrich 